\title{
Some Regularity Criteria for the 3D Boussinesq Equations in the Class $L^{2}\left(0, T ; \dot{B}_{\infty, \infty}^{-1}\right)$
}

\author{
Zujin Zhang \\ School of Mathematics and Computer Science, Gannan Normal University, Ganzhou 341000, China \\ Correspondence should be addressed to Zujin Zhang; zhangzujin361@163.com
}

Received 2 December 2013; Accepted 15 January 2014; Published 23 February 2014

Academic Editors: Y. Dimakopoulos and X.-S. Yang

Copyright (C) 2014 Zujin Zhang. This is an open access article distributed under the Creative Commons Attribution License, which permits unrestricted use, distribution, and reproduction in any medium, provided the original work is properly cited.

We consider the three-dimensional Boussinesq equations and obtain some regularity criteria via the velocity gradient (or the vorticity, or the deformation tensor) and the temperature gradient.

\section{Introduction}

Consider the following three-dimensional (3D) Boussinesq equations:

$$
\begin{gathered}
\mathbf{u}_{t}+(\mathbf{u} \cdot \nabla) \mathbf{u}-\Delta \mathbf{u}+\nabla \pi=\theta \mathbf{e}_{3}, \\
\theta_{t}+(\mathbf{u} \cdot \nabla) \theta-\Delta \theta=0, \\
\nabla \cdot \mathbf{u}=0, \\
\mathbf{u}(x, 0)=\mathbf{u}_{0}, \quad \theta(x, 0)=\theta_{0} .
\end{gathered}
$$

Here, $\mathbf{u}=\left(u_{1}(x, t), u_{2}(x, t), u_{3}(x, t)\right)$ is the fluid velocity, $\pi=\pi(x, t)$ is a scalar pressure, and $\theta=\theta(x, t) \geq 0$ is the temperature, while $\mathbf{u}_{0}$ and $\theta_{0}$ are the prescribed initial velocity and temperature, respectively.

When $\theta=0$, (1) reduces to the incompressible NavierStokes equations. The regularity of its weak solutions and the existence of global strong solutions are challenging open problems; see [1-3]. Initialed by $[4,5]$, there have been a lot of literatures devoted to finding sufficient conditions to ensure the smoothness of the solutions; see [6-18] and so forth. Since the convective terms are similar in Navier-Stokes equations and Boussinesq equations, the authors also consider the regularity conditions for (1); see [19-23] and so forth.

Motivated by [7], we will consider the regularity criteria for (1) and extend the result of [7] to the case of Boussinesq equations.

Before stating the precise result, let us recall the weak formulation of (1).
Definition 1. Let $\mathbf{u}_{0} \in L^{2}\left(\mathbb{R}^{3}\right), \theta_{0} \in L^{1} \cap L^{\infty}\left(\mathbb{R}^{3}\right)$, and $T>0$. A measurable pair $(\mathbf{u}, \theta)$ is said to be a weak solution of (1) in $(0, T)$, provided that

(1) $(\mathbf{u}, \theta) \in L^{\infty}\left(0, T ; L^{2}\left(\mathbb{R}^{3}\right)\right) \cap L^{2}\left(0, T ; H^{1}\left(\mathbb{R}^{3}\right)\right), \theta \in$ $L^{\infty}\left(0, T ; L^{1} \cap L^{\infty}\left(\mathbb{R}^{3}\right)\right)$;

(2) (1) $)_{1,2,3}$ are satisfied in the sense of distributions;

(3) the energy inequality

$$
\|(\mathbf{u}, \theta)\|_{L^{2}}^{2}+2 \int_{0}^{t}\|\nabla(\mathbf{u}, \theta)\|_{L^{2}}^{2} \mathrm{~d} s \leq \int_{0}^{t} \int_{\mathbb{R}^{3}} \theta u_{3} \mathrm{~d} x \mathrm{~d} s,
$$

for all $0 \leq t \leq T$.

Now, our main result reads the following.

Theorem 2. Let $\mathbf{u}_{0} \in L^{2}\left(\mathbb{R}^{3}\right)$ with $\nabla \cdot \mathbf{u}_{0}=0$ in the sense of distributions, $\theta_{0} \in L^{1} \cap L^{\infty}\left(\mathbb{R}^{3}\right)$. Suppose that $(\mathbf{u}, \theta)$ is a weak solution of (1) in $[0, T)$, and

$$
(\nabla \mathbf{u}, \nabla \theta) \in L^{2}\left(0, T ; \dot{B}_{\infty, \infty}^{-1}\left(\mathbb{R}^{3}\right)\right) ;
$$

then the solution $(\mathbf{u}, \theta) \in C^{\infty}\left((0, T) \times \mathbb{R}^{3}\right)$.

Due to the divergence-free condition of the fluid velocity u, we have

$$
\Delta u_{i}=\sum_{k=1}^{3} \partial_{k}\left(\partial_{k} u_{i}-\partial_{i} u_{k}\right), \quad \Delta u_{i}=\sum_{k=1}^{3} \partial_{k}\left(\partial_{k} u_{i}+\partial_{i} u_{k}\right)
$$


Thus,

$$
\begin{aligned}
& \partial_{j} u_{i}=-\sum_{k=1}^{3} \mathscr{R}_{j} \mathscr{R}_{k}\left(\partial_{k} u_{i}-\partial_{i} u_{k}\right), \\
& \partial_{j} u_{i}=-\sum_{k=1}^{3} \mathscr{R}_{j} \mathscr{R}_{k}\left(\partial_{k} u_{i}+\partial_{i} u_{k}\right) .
\end{aligned}
$$

Here, $\mathscr{R}_{j}=\partial_{j} / \sqrt{-\Delta}$ is the Riesz transformation.

Using (5), we can deduce easily from Theorem 2 the following.

Corollary 3. Let $\mathbf{u}_{0} \in L^{2}\left(\mathbb{R}^{3}\right)$ with $\nabla \cdot \mathbf{u}_{0}=0$ in the sense of distributions, $\theta_{0} \in L^{1} \cap L^{\infty}\left(\mathbb{R}^{3}\right)$. Suppose that $(\mathbf{u}, \theta)$ is a weak solution of (1) in $[0, T)$, and

$$
(\nabla \times \mathbf{u}, \nabla \theta) \in L^{2}\left(0, T ; \dot{B}_{\infty, \infty}^{-1}\left(\mathbb{R}^{3}\right)\right),
$$

or

$$
(\operatorname{Def} \mathbf{u}, \nabla \theta) \in L^{2}\left(0, T ; \dot{B}_{\infty, \infty}^{-1}\left(\mathbb{R}^{3}\right)\right)
$$

then the solution $(\mathbf{u}, \theta) \in C^{\infty}\left((0, T) \times \mathbb{R}^{3}\right)$. Here, $\nabla \times \mathbf{u}=$ $\left|\begin{array}{ccc}i & j & k \\ \partial_{1} & \partial_{2} & \partial_{3} \\ u_{1} & u_{2} & u_{3}\end{array}\right|$ is the vorticity and (Def $\left.\mathbf{u}\right)_{i j}=\partial_{i} u_{j}+\partial_{j} u_{i}$ is the deformation tensor (the symmetric tensor of the rate of strain).

The rest of this paper is organized as follows. In Section 2, we recall the definition of Besov spaces and the related interpolation inequalities. Section 3 is devoted to proving Theorem 2.

\section{Preliminaries}

We first introduce the Littlewood-Paley decomposition. Let $S\left(\mathbb{R}^{3}\right)$ be the Schwartz class of rapidly decreasing functions. For $f \in \mathcal{S}\left(\mathbb{R}^{3}\right)$, its Fourier transform $\mathscr{F} f=\widehat{f}$ is defined as

$$
\widehat{f}(\xi)=\int_{\mathbb{R}^{3}} f(x) e^{-i x \cdot \xi} \mathrm{d} x .
$$

Let us choose a nonnegative radial function $\varphi \in \mathcal{S}\left(\mathbb{R}^{3}\right)$ such that

$$
0 \leq \widehat{\varphi}(\xi) \leq 1, \quad \widehat{\varphi}(\xi)= \begin{cases}1, & \text { if }|\xi| \leq 1 \\ 0, & \text { if }|\xi| \geq 2\end{cases}
$$

and let

$$
\begin{array}{r}
\psi(x)=\varphi(x)-2^{-3} \varphi\left(\frac{x}{2}\right), \\
\varphi_{j}(x)=2^{3 j} \varphi\left(2^{j} x\right), \\
\psi_{j}(x)=2^{3 j} \psi\left(2^{j} x\right), \quad j \in \mathbb{Z} .
\end{array}
$$

For $j \in \mathbb{Z}$, the Littlewood-Paley projection operators $S_{j}$ and $\Delta_{j}$ are, respectively, defined by

$$
S_{j} f=\varphi_{j} * f, \quad \Delta_{j} f=\psi_{j} * f .
$$

Observe that $\Delta_{j}=S_{j}-S_{j-1}$. Also, it is easy to check that if $f \in L^{2}\left(\mathbb{R}^{3}\right)$, then

$$
\begin{aligned}
& S_{j} f \longrightarrow 0, \quad \text { as } j \longrightarrow-\infty ; \\
& S_{j} f \longrightarrow f, \quad \text { as } j \longrightarrow \infty,
\end{aligned}
$$

in the $L^{2}$ sense. By telescoping the series, we have the following Littlewood-Paley decomposition:

$$
f=\sum_{j=-\infty}^{\infty} \Delta_{j} f
$$

for all $f \in L^{2}\left(\mathbb{R}^{3}\right)$, where the summation is in the $L^{2}$ sense.

Let $s \in \mathbb{R} ; p, q \in[1, \infty]$; the homogeneous Besov space $\dot{B}_{p, q}^{s}\left(\mathbb{R}^{3}\right)$ is defined by the full dyadic decomposition such as

$$
\dot{B}_{p, q}^{s}=\left\{f \in \mathscr{Z}^{\prime}\left(\mathbb{R}^{3}\right) ;\|f\|_{\dot{B}_{p, q}^{s}}=\left\|\left\{2^{j s}\left\|\Delta_{j} f\right\|_{L^{p}}\right\}_{j=-\infty}^{\infty}\right\|_{\ell^{q}}<\infty\right\},
$$

where $\mathscr{Z}^{\prime}\left(\mathbb{R}^{3}\right)$ is the dual space of

$$
\mathscr{Z}\left(\mathbb{R}^{3}\right)=\left\{f \in \mathcal{S}\left(\mathbb{R}^{3}\right) ; D^{\alpha} \widehat{f}(0)=0, \forall \alpha \in \mathbb{N}^{3}\right\} .
$$

The following interpolation inequality will be needed in Section 3:

$$
\|f\|_{L^{4}} \leq C\|f\|_{\dot{H}^{1}}^{1 / 2}\|f\|_{\dot{B}_{\infty, \infty}^{-1}}^{1 / 2}, \quad \forall f \in \dot{H}^{1}\left(\mathbb{R}^{3}\right) \cap \dot{B}_{\infty, \infty}^{-1}\left(\mathbb{R}^{3}\right)
$$

See [24] for the proof.

\section{Proof of Theorem 2}

In this section, we will prove Theorem 2 .

Multiplying (1) by $-\Delta \mathbf{u}$ and $(1)_{2}$ by $-\Delta \theta$, integrating over $\mathbb{R}^{3}$, and summing up, we obtain

$$
\begin{aligned}
\frac{1}{2} \frac{\mathrm{d}}{\mathrm{d} t} \| & \nabla(\mathbf{u}, \theta)\left\|_{L^{2}}^{2}+\right\| \Delta(\mathbf{u}, \theta) \|_{L^{2}}^{2} \\
= & \int_{\mathbb{R}^{3}}[(\mathbf{u} \cdot \nabla) \mathbf{u}] \cdot \Delta \mathbf{u} \mathrm{d} x-\int_{\mathbb{R}^{3}} \theta \Delta u_{3} \mathrm{~d} x \\
& +\int_{\mathbb{R}^{3}}[(\mathbf{u} \cdot \nabla) \theta] \cdot \Delta \theta \mathrm{d} x \\
= & -\sum_{i, j, k=1}^{3} \int_{\mathbb{R}^{3}} \partial_{k} u_{j} \partial_{j} u_{i} \partial_{k} u_{i} \mathrm{~d} x \\
& +\int_{\mathbb{R}^{3}} \nabla \theta \cdot \nabla u_{3} \mathrm{~d} x-\sum_{j, k=1}^{3} \int_{\mathbb{R}^{3}} \partial_{k} u_{j} \partial_{j} \theta \partial_{k} \theta \mathrm{d} x \\
\equiv & I_{1}+I_{2}+I_{3} .
\end{aligned}
$$


Invoking Hölder inequality, (17), and Young inequality, we may bound $I_{1}$ as

$$
\begin{aligned}
I_{1} & \leq\|\nabla \mathbf{u}\|_{L^{4}}^{2}\|\nabla \mathbf{u}\|_{L^{2}} \\
& \leq C\|\nabla \mathbf{u}\|_{\dot{B}_{\infty, \infty}^{-1}}\|\Delta \mathbf{u}\|_{L^{2}}\|\nabla \mathbf{u}\|_{L^{2}} \\
& \leq C\|\nabla \mathbf{u}\|_{\dot{B}_{\infty, \infty}^{-1}}^{2}\|\nabla \mathbf{u}\|_{L^{2}}^{2}+\frac{1}{2}\|\Delta \mathbf{u}\|_{L^{2}}^{2} .
\end{aligned}
$$

For $I_{2}$, we use Hölder inequality to dominate as

$$
I_{2} \leq \frac{1}{2} \int_{\mathbb{R}^{3}}\left(|\nabla \theta|^{2}+|\nabla \mathbf{u}|^{2}\right) \mathrm{d} x=\frac{1}{2}\left(\|\nabla \theta\|_{L^{2}}^{2}+\|\nabla \mathbf{u}\|_{L^{2}}^{2}\right) .
$$

Finally, $I_{3}$ can be estimated similarly as $I_{1}$,

$$
\begin{aligned}
I_{3} & \leq\|\nabla \theta\|_{L^{4}}^{2}\|\nabla \mathbf{u}\|_{L^{2}} \\
& \leq C\|\nabla \theta\|_{\dot{B}_{\infty, \infty}^{-1}}\|\Delta \theta\|_{L^{2}}\|\nabla \mathbf{u}\|_{L^{2}} \\
& \leq C\|\nabla \theta\|_{\dot{B}_{\infty, \infty}^{-1}}^{2}\|\nabla \mathbf{u}\|_{L^{2}}^{2}+\frac{1}{2}\|\Delta \theta\|_{L^{2}}^{2} .
\end{aligned}
$$

Substituting (19), (20), and (21) into (18), we gather

$$
\begin{aligned}
& \frac{\mathrm{d}}{\mathrm{d} t}\|\nabla(\mathbf{u}, \theta)\|_{L^{2}}^{2}+\|\Delta(\mathbf{u}, \theta)\|_{L^{2}}^{2} \\
& \quad \leq C\left(\|\nabla(\mathbf{u}, \theta)\|_{\dot{B}_{\infty, \infty}^{-1}}^{2}+1\right)\|\nabla(\mathbf{u}, \theta)\|_{L^{2}}^{2} .
\end{aligned}
$$

Gronwall inequality together with (3) then implies that

$$
(\mathbf{u}, \theta) \in L^{\infty}\left(0, T ; H^{1}\left(\mathbb{R}^{3}\right)\right) \cap L^{2}\left(0, T ; H^{2}\left(\mathbb{R}^{3}\right)\right) .
$$

Then, we may use standard energy method to drive highorder derivative bounds, which would imply $(\mathbf{u}, \theta) \in$ $C^{\infty}\left((0, T) \times \mathbb{R}^{3}\right)$ by Sobolev embedding theorems, as desired.

The proof of Theorem 2 is completed.

\section{Conflict of Interests}

The author declares that there is no conflict of interests regarding the publication of this paper.

\section{Acknowledgments}

Zujin Zhang was partially supported by the Youth Natural Science Foundation of Jiangxi Province (20132BAB211007) and the National Natural Science Foundation of China (11326138).

\section{References}

[1] E. Hopf, "Über die Anfangswertaufgabe für die hydrodynamischen Grundgleichungen," Mathematische Nachrichten, vol. 4, pp. 213-231, 1951.

[2] P. G. Lemarié-Rieusset, Recent Developments in the NavierStokes Problem, Chapman \& Hall/CRC Research Notes in Mathematics, Chapman \& Hall/CRC, Boca Raton, Fla, USA, 2002.
[3] J. Leray, "Sur le mouvement d'un liquide visqueux emplissant l'espace," Acta Mathematica, vol. 63, no. 1, pp. 193-248, 1934.

[4] J. Serrin, "On the interior regularity of weak solutions of the Navier-Stokes equations," Archive for Rational Mechanics and Analysis, vol. 9, pp. 187-191, 1962.

[5] J. Serrin, "The initial value problem for the Navier-Stokes equations," in Nonlinear Problems, pp. 69-98, University of Wisconsin Press, Madison, Wis, USA, 1963.

[6] H. B. da Veiga, "A new regularity class for the Navier-Stokes equations in $\mathbb{R}^{n}$," Chinese Annals of Mathematics $B$, vol. 16 , no. 4, pp. 407-412, 1995.

[7] S. Gala, "A remark on the blow-up criterion of strong solutions to the Navier-Stokes equations," Applied Mathematics and Computation, vol. 217, no. 22, pp. 9488-9491, 2011.

[8] X. W. He and S. Gala, "Regularity criterion for weak solutions to the Navier-Stokes equations in terms of the pressure in the class $L^{2}\left(0, T ; \dot{B}_{\infty, \infty}^{-1}\left(\mathbb{R}^{3}\right)\right)$," Nonlinear Analysis: Real World Applications, vol. 12, no. 6, pp. 3602-3607, 2011.

[9] J. Neustupa, A. Novotný, and P. Penel, "An interior regularity of a weak solution to the Navier-Stokes equations in dependence on one component of velocity," in Topics in Mathematical Fluid Mechanics, vol. 10 of Quaderni di Matematica, pp. 163-183, 2002.

[10] Z. J. Zhang, "A logarithmically improved regularity criterion for the $3 \mathrm{D}$ Boussinesq equations via the pressure," Acta Applicandae Mathematicae, 2013.

[11] Z. J. Zhang, "A remark on the regularity criterion for the 3D Navier-Stokes equations involving the gradient of one velocity component," Journal of Mathematical Analysis and Applications, 2014.

[12] Z. J. Zhang, "A Serrin-type regularity criterion for the NavierStokes equations via one velocity component," Communications on Pure and Applied Analysis, vol. 12, no. 1, pp. 117-124, 2013.

[13] Z. J. Zhang, P. Li, and D. X. Zhong, "Navier-Stokes equations with regularity in two entries of the velocity gradient tensor," Applied Mathematics and Computation, vol. 228, pp. 546-551, 2014.

[14] Z. J. Zhang, Z. A. Yao, P. Li, C. C. Guo, and M. Lu, "Two new regularity criteria for the 3D Navier-Stokes equations via two entries of the velocity gradient tensor," Acta Applicandae Mathematicae, vol. 123, pp. 43-52, 2013.

[15] Z. J. Zhang, D. X. Zhong, and L. Hu, "A new regularity criterion for the 3D Navier-Stokes equations via two entries of the velocity gradient tensor," Acta Applicandae Mathematicae, vol. 129, no. 1, pp. 175-181, 2014.

[16] Y. Zhou, "A new regularity criterion for weak solutions to the Navier-Stokes equations," Journal de Mathématiques Pures et Appliquées, vol. 84, no. 11, pp. 1496-1514, 2005.

[17] Y. Zhou and M. Pokorný, "On a regularity criterion for the Navier-Stokes equations involving gradient of one velocity component," Journal of Mathematical Physics, vol. 50, no. 12, Article ID 123514, 11 pages, 2009.

[18] Y. Zhou and M. Pokorný, "On the regularity of the solutions of the Navier-Stokes equations via one velocity component," Nonlinearity, vol. 23, no. 5, pp. 1097-1107, 2010.

[19] J. S. Fan and Y. Zhou, "A note on regularity criterion for the 3D Boussinesq system with partial viscosity," Applied Mathematics Letters, vol. 22, no. 5, pp. 802-805, 2009.

[20] N. Ishimura and H. Morimoto, "Remarks on the blow-up criterion for the 3-D Boussinesq equations," Mathematical Models \& Methods in Applied Sciences, vol. 9, no. 9, pp. 13231332, 1999. 
[21] H. Qiu, Y. Du, and Z. Yao, "Blow-up criteria for 3D Boussinesq equations in the multiplier space," Communications in Nonlinear Science and Numerical Simulation, vol. 16, no. 4, pp. 18201824, 2011.

[22] H. Qiu, Y. Du, and Z. Yao, "Serrin-type blow-up criteria for 3D Boussinesq equations," Applicable Analysis, vol. 89, no. 10, pp. 1603-1613, 2010.

[23] Z. Zhang, "A remark on the regularity criterion for the 3D Boussinesq equations involving the pressure gradient," Abstract and Applied Analysis, vol. 2014, Article ID 510924, 4 pages, 2014.

[24] Y. Meyer, P. Gerard, and F. Oru, "Inégalités de Sobolev précisées," in Séminaire sur les Équations aux Dérivées Partielles, 1996-1997, Exp. No. 4, p. 8, École Polytechnique, Palaiseau, France, 1997. 


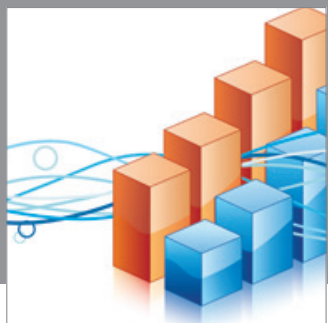

Advances in

Operations Research

mansans

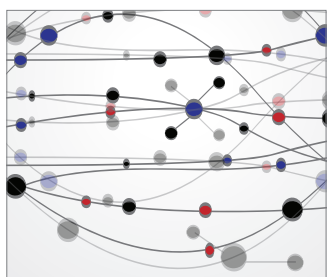

The Scientific World Journal
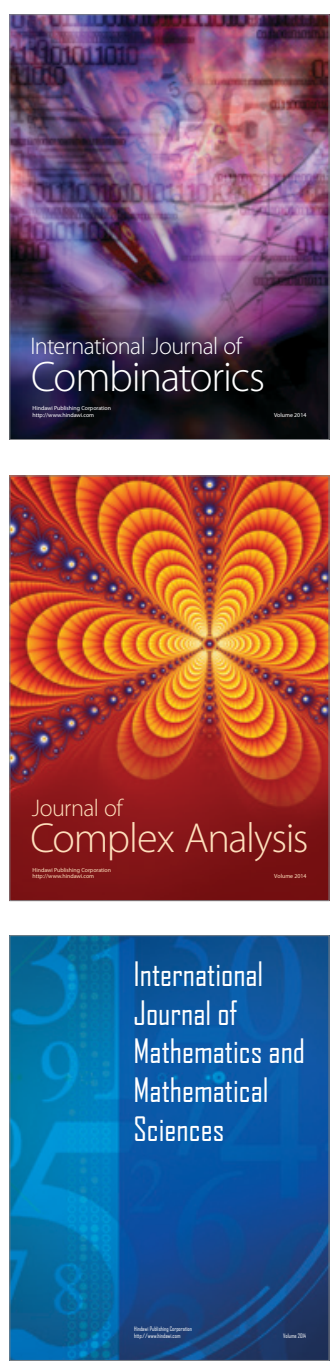
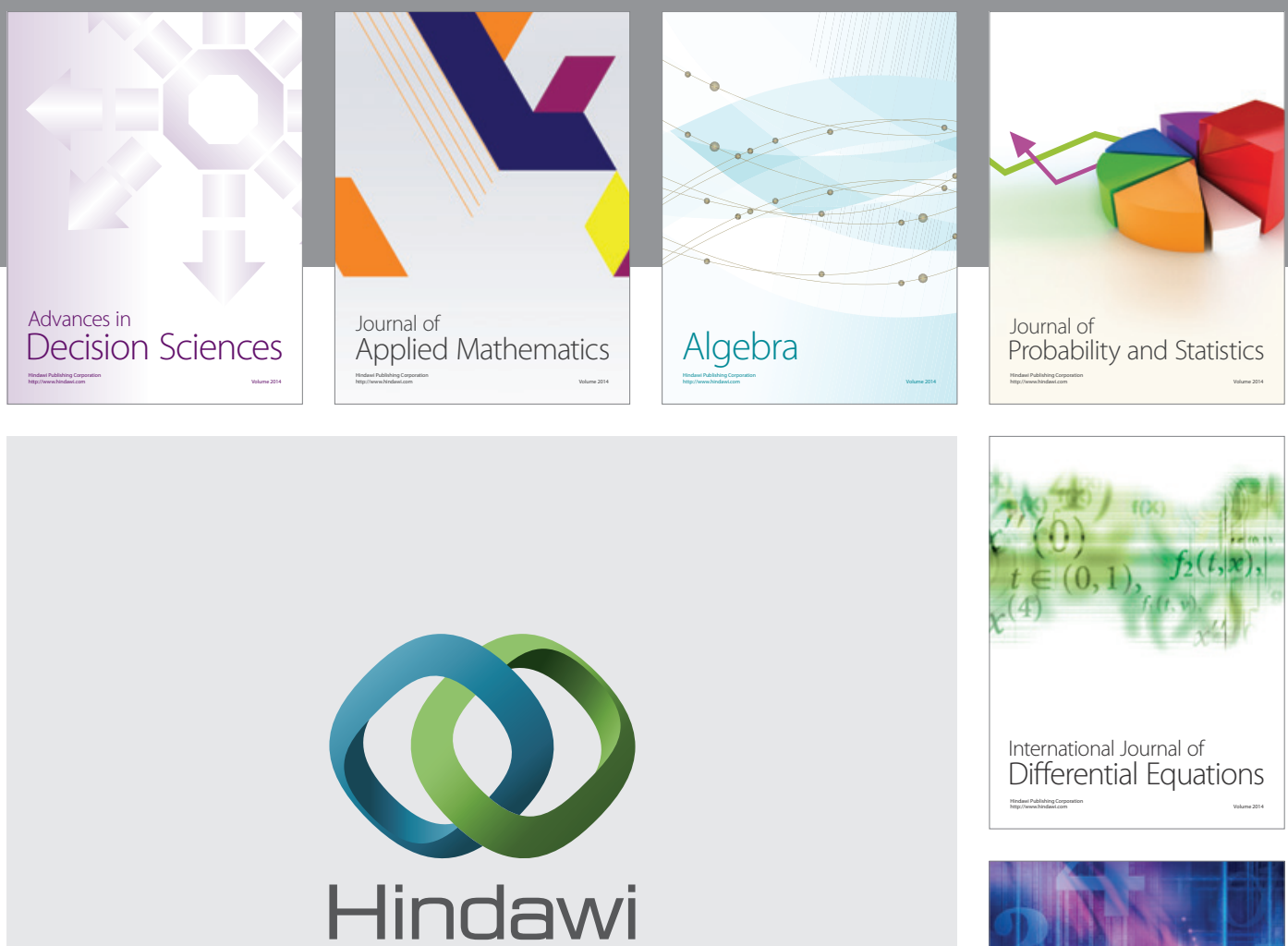

Submit your manuscripts at http://www.hindawi.com
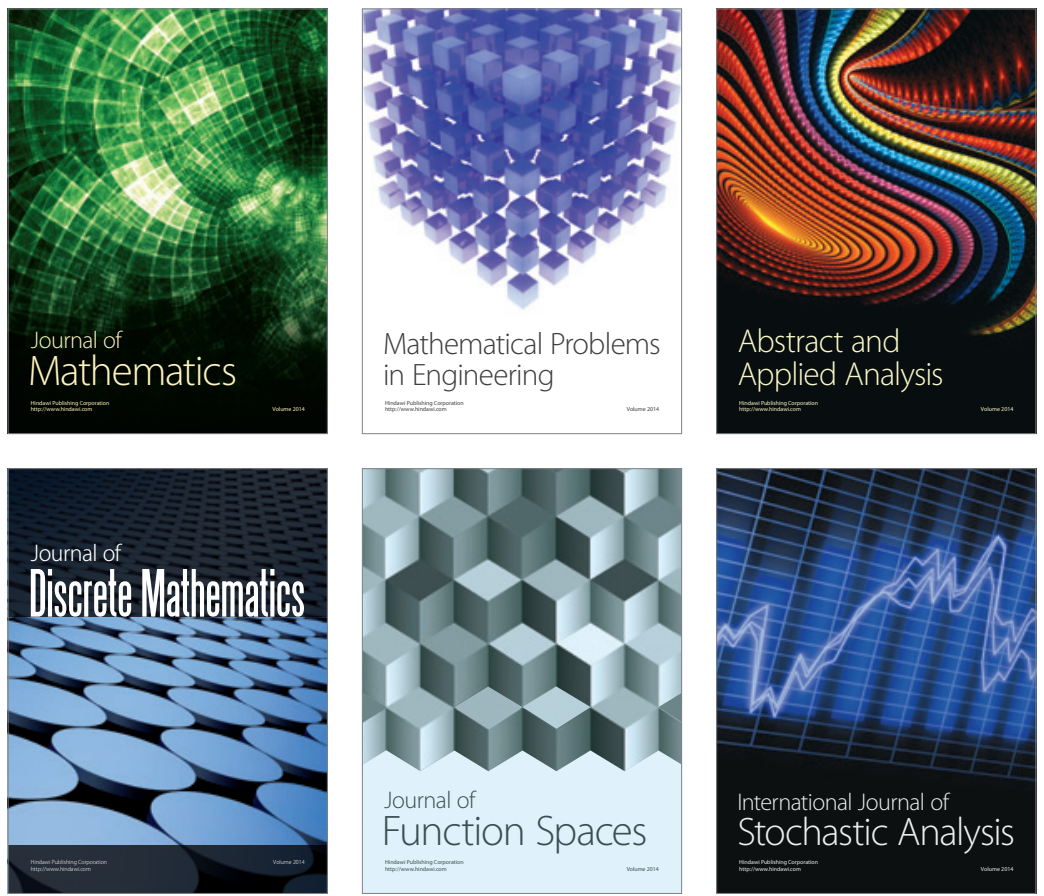

Journal of

Function Spaces

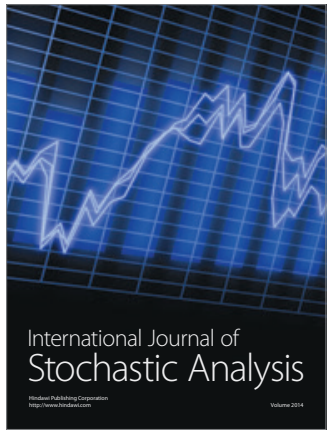

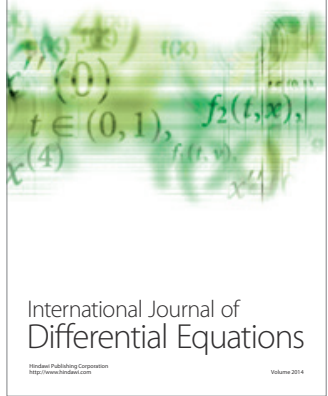
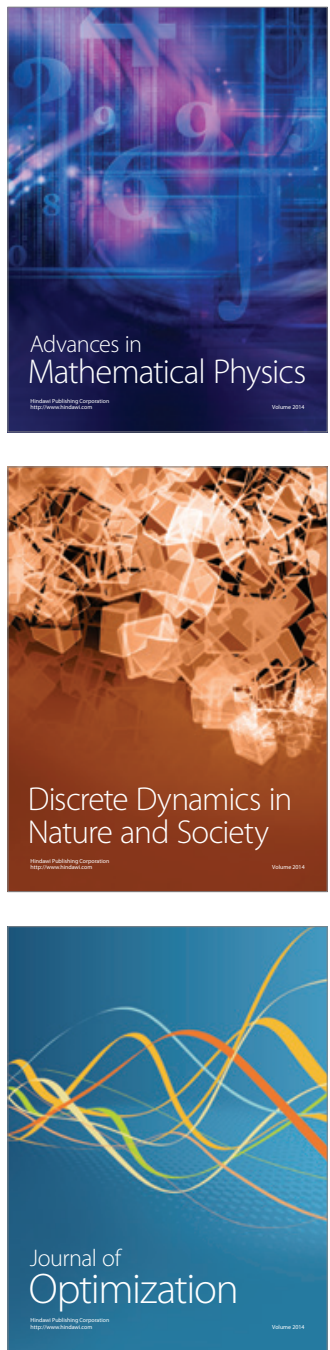